University of New Hampshire

University of New Hampshire Scholars' Repository

Space Science Center

Institute for the Study of Earth, Oceans, and

Space (EOS)

1993

\title{
COMPTEL gamma ray and neutron measurements of solar flares
}

\author{
James M. Ryan \\ University of New Hampshire, James.Ryan@unh.edu \\ D J. Forrest \\ University of New Hampshire - Main Campus \\ J Lockwood \\ University of New Hampshire - Main Campus \\ M Loomis \\ University of New Hampshire - Main Campus \\ Mark L. McConnell \\ University of New Hampshire - Main Campus, mark.mcconnell@unh.edu
}

See next page for additional authors

Follow this and additional works at: https://scholars.unh.edu/ssc

Part of the Astrophysics and Astronomy Commons

\section{Recommended Citation}

COMPTEL gamma ray and neutron measurements of solar flares Ryan, J. and Forrest, D. and Lockwood, J. and Loomis, M. and McConnell, M. and Morris, D. and Webber, W. and Rank, G. and Schönfelder, V. and Swanenburg, B. N. and Bennett, K. and Hanlon, L. and Winkler, C. and Debrunner, H., AIP Conference Proceedings, 280, 631-642 (1993), DOl:http://dx.doi.org/10.1063/1.44245

This Conference Proceeding is brought to you for free and open access by the Institute for the Study of Earth, Oceans, and Space (EOS) at University of New Hampshire Scholars' Repository. It has been accepted for inclusion in Space Science Center by an authorized administrator of University of New Hampshire Scholars' Repository. For more information, please contact Scholarly.Communication@unh.edu. 


\section{Authors}

James M. Ryan, D J. Forrest, J Lockwood, M Loomis, Mark L. McConnell, D Morris, W Webber, G Rank, V Schonfelder, B Swanenburg, K Bennett, L O. Hanlon, C Winkler, and H Debrunner 


\section{AIP $\mid$ Proceedings}

\section{COMPTEL gamma ray and neutron measurements of solar}

\section{flares}

J. Ryan, D. Forrest, J. Lockwood, M. Loomis, M. McConnell, D. Morris, W. Webber

, G. Rank, V. Schönfelder, B. N. Swanenburg, K. Bennett, L. Hanlon, C. Winkler, and H. Debrunner

Citation: AIP Conference Proceedings 280, 631 (1993); doi: 10.1063/1.44245

View online: http://dx.doi.org/10.1063/1.44245

View Table of Contents:

http://scitation.aip.org/content/aip/proceeding/aipcp/280?ver=pdfcov

Published by the AIP Publishing

\section{Articles you may be interested in}

Neutron and gammaray measurements of the solar flare of 1991 June 9

AIP Conf. Proc. 294, 89 (1994); 10.1063/1.45205

Observations of the 1991 June 11 solar flare with COMPTEL

AIP Conf. Proc. 280, 661 (1993); 10.1063/1.44249

High energy processes in solar flares

AIP Conf. Proc. 280, 643 (1993); 10.1063/1.44246

OSSE observations of solar flares

AIP Conf. Proc. 280, 619 (1993); 10.1063/1.44244

About the ability of GRASP to measure diffuse gammaray line sources

AIP Conf. Proc. 170, 427 (1988); 10.1063/1.37241 
J. Ryan, D. Forrest, J. Lockwood, M. Loomis, M. McConnell, D, Morris, W. Webber Space Science Center, Morse Hall, University of New Hampshire, Durham, NH 03824

G. Rank, V. Schönfelder

Max-Planck Institut für Extraterrestrische Physik, 8046 Garching, Germany

B.N. Swanenburg

SRON-Leiden, 2300 RALeiden, The Netherlands

K. Bennett, L. Hanlon, C. Winkler

Astrophysics Divsion, European Space Research and Technology Center, Noordwijk, The Netherlands

H. Debrunner

University of Bern, Bern, Switzerland

ABSTRACT

COMPTEL on the Compton Gamma Ray Observatory has measured the flux of $\gamma$-rays and neutrons from several solar flares. These data have also been used to image the Sun in both forms of radiation. Unusually intense flares occurred during June 1991 yielding data sets that offer some new insight into of how energetic protons and electrons are accelerated and behave in the solar environment. We summarize here some of the essential features in the solar flare data as obtained by COMPTEL during June 1991.

\section{INTRODUCTION}

COMPTEL on the Compton Gamma Ray Observatory (CGRO) was designed to measure the flux of cosmic gamma ray sources down to a level of $\sim 10^{-5} \gamma-\mathrm{cm}^{-2}-\mathrm{s}^{-1}-\mathrm{MeV}^{-1}$ in the energy range of $1-30 \mathrm{MeV}$. However, it is also capable of measuring the $\gamma$-ray and neutron flux from solar flares. Although the intensity of large solar flares exceeds the dynamic range of the telescope, useful measurements still can be performed even for the most intense events. Such was the case in June 1991 when active region 6659 was declared to be a CGRO Target of Opportunity and the capabilities of the observatory were directed toward making the highest quality measurements of flares during the transit of the active region across the solar disk.

From the emergence of the region on the east limb on 1 June until 8 June 1991 the Sun was outside the field-of-view of COMPTEL. From 8 June until passage of the region around the west limb on 15 June, the Sun was in the telescope field-ofview at a zenith angle of $\sim 10^{\circ}$ to $15^{\circ}$. Other periods of solar activity have produced solar flares that COMPTEL measured; however, we report here on the more spectacular events seen in June 1991, including those from active region 6659 . The flare data summarized in this paper are from events on 4, 9, 11, 15 and 30 June 1991. Only the flares of 9, 11 and 15 June 1991 were within the COMPTEL field-of-view.

The design of COMPTEL is illustrated schematically in Figure 1. COMPTEL has two modes of measuring $\gamma$-rays from cosmic bursts and solar flares ${ }^{1}$. We only 
present here the salient features of the telescope operation as they relate to solar measurements. The two methods of $\gamma$-ray detection are the so-called burst mode and telescope mode.

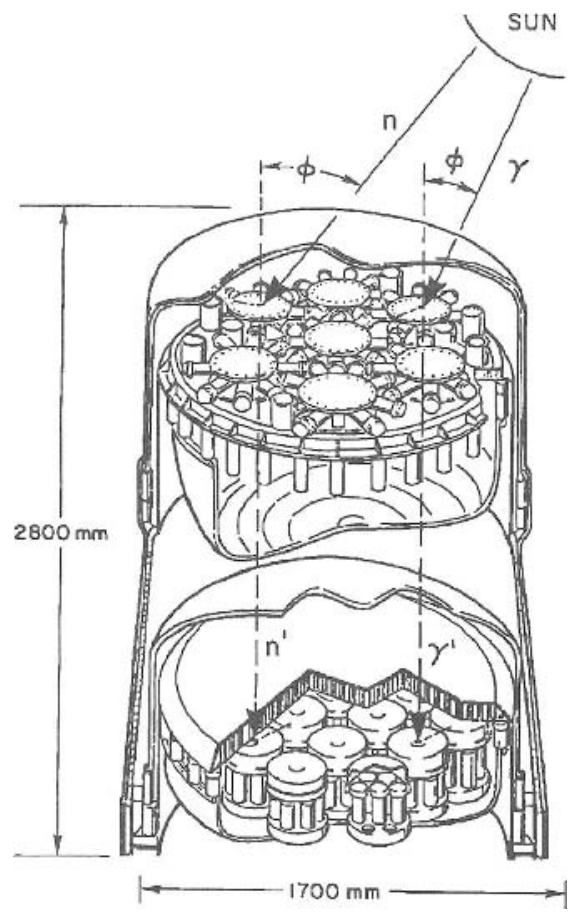

COMPTEL

IMAGING COMPTON TELESCOPE

In the telescope mode an incoming $\gamma$-ray scatters off an electron in one of seven D1 detectors and proceeds down to one of fourteen D2 detectors scattering again. Events fully absorbed in D2 constitute the ideal type of $\gamma$-ray interaction. The material in D1 is a liquid organic scintillator NE213A with the properties of low density and low $Z(\mathrm{H} / \mathrm{C}$ ratio $=1.286)$. The material scatters both $\gamma$-rays and neutrons elastically, off electrons and hydrogen nuclei respectively. The scatters take place according to the Compton kinematic formula

$$
\begin{aligned}
\phi= & \cos ^{-1}\left\{\left(1-\varepsilon / E_{2}\right.\right. \\
& \left.\left.+\varepsilon /\left(E_{1}+E_{2}\right)\right)\right\},
\end{aligned}
$$

where $\varepsilon$ is the electron rest mass energy, $E_{1}$ is the energy deposit in $D 1, E_{2}$ is the energy deposit in D2 and $\phi$ is the Compton scatter angle provided $E_{1}+E_{2}$ is the full incident $\gamma$-ray energy.

As an imaging solar $\gamma$-ray telescope in the energy range of 0.8 $30 \mathrm{MeV}$, COMPTEL relies on the full energy deposit of the $\gamma$-ray to correctly estimate the scattering angle $\phi$ of the photon within the instrument. For a solar flare $\gamma$-ray interacting in COMPTEL, the

Figure 1. Schematic of COMPTEL with typical $\gamma$-ray and neutron interactions. inferred scatter angle $\phi$ about the vector of the scattered $\gamma$-ray must be such that the photon is assigned a solar origin as indicated schematically in Figure 1. Hence, we know that the photon deposited its full energy in the detector. The response of the telescope to such events is simple. The energy or pulse height distribution is Gaussian in shape, but with a heavily suppressed Compton tail at low energies. Since the solar $\gamma$-ray spectra are rich in lines from $\mathrm{C}, \mathrm{N}, \mathrm{O}, \mathrm{Ne}, \mathrm{Mg}$ etc., a simple instrumental response function facilitates correct de-convolution of the pulse height spectra.

The liquid organic scintillator in D1 possesses pulse shape discrimination (PSD) properties, in that energetic protons produce light pulses with longer rise times than those of electrons (and other minimum ionizing particles). This capability allows for efficient identification of signals from recoil protons produced by fast neutrons scattering elastically off hydrogen in D1.

The D1 and D2 subsystems of the telescope are each completely surrounded by charged particle detectors (see Fig. 1). These 4 domes of plastic scintillator NE110 
are $1.5 \mathrm{~cm}$ thick and do not significantly attenuate the incident energetic $\gamma$-ray or neutron fluxes, yet are virtually $100 \%$ efficient in identifying charged cosmic rays. The charged particle shields and other intervening material heavily attenuate the solar flare hard X-ray flux, minimizing pulse pile-up effects in the D1 and D2 detectors. They do, however, saturate under the intense soft thermal X-ray flux associated with flares. The effect of this is seen below when we discuss the behavior of the telescope during intense events.

Neutrons are also measured in the telescope mode. The ideal type of neutron interaction in COMPTEL occurs when the incoming neutron scatters elastically off a hydrogen nucleus in the D1 detector. The scattered neutron then proceeds to the D2 detector where it may interact, depositing some of its energy to produce a trigger signal as indicated in Figure 1. The energy of the incident neutron is computed by summing the proton recoil energy $E_{1}$ in the D1 detector with the energy of the scattered neutron $E_{S}$ deduced from the time-of-flight (TOF) from the D1 to the D2 detector. The scatter angle for non-relativistic neutrons $(<150 \mathrm{MeV})$ can be computed by the formula:

$$
\tan ^{2} \phi=E_{1} / E_{s} .
$$

As with $\gamma$-rays, neutrons can be traced backwards from $D 2$ to $D 1$ through the angle $\phi$ to a cone mantle restricting the incident direction to include the Sun. This is a geometrical constraint identical to that of the $\gamma$-ray measurements. The pulse shape from recoil protons is sufficiently different from that of electrons to reject more than $95 \%$ of electron-recoil events greater than about $1 \mathrm{MeV}$, the energy threshold in D1 for neutron detection. The PSD and TOF criteria in this channel are set such that solar neutrons incident on D1 in the energy range from about $10 \mathrm{MeV}$ to $150 \mathrm{MeV}$ are recorded. In this energy interval COMPTEL can observe neutrons from about 14.5 to 55 minutes after release from the Sun. This corresponds to a minimum observed delay time of 6 to 47 minutes after the onset of the $\gamma$-ray flash (assuming neutrons are not produced without accompanying $\gamma$-rays).

The burst mode of the COMPTEL instrument can also be used to detect solar $\gamma$-rays ${ }^{2}$. One D2 detector module covers the energy interval from 0.1 to $1 \mathrm{MeV}$ and another the interval from $\sim 1$ to $10 \mathrm{MeV}$. Each detector module has an unobscured field-of-view of about $2.5 \mathrm{sr}$ and a physical area of $\sim 600 \mathrm{~cm}^{2}$. Outside this field-ofview varying amounts of intervening material exist attenuating the solar $\gamma$-ray flux.

\section{OBSERVATIONS}

\section{June 1991}

This $\mathrm{X} 12+/ 3 \mathrm{~B}$ flare occurred at coordinates N30E70. The X-ray flare started at 0337 UT. At this time the Sun was $\sim 105^{\circ}$ from the COMPTEL axis, well outside the telescope field-of-view. The following data come only from the high range burst detector operating from $\sim 600 \mathrm{keV}$ to $\sim 10 \mathrm{MeV}$. The low energy range burst detector was not operating at this time. The intensity-time profile of the burst detector count rate $>600 \mathrm{keV}$ is shown in Figure 2 .

The burst spectrometer was activated by the trigger signal provided by BATSE at 0337 UT. The peak intensity occurred at 0341:15 UT as seen in the large reduction in the count rate produced by dead time effects. The flare continued up to at least 0350 UT at which time the burst spectrometer reverted back to its low time resolution (100 s) background cadence. 


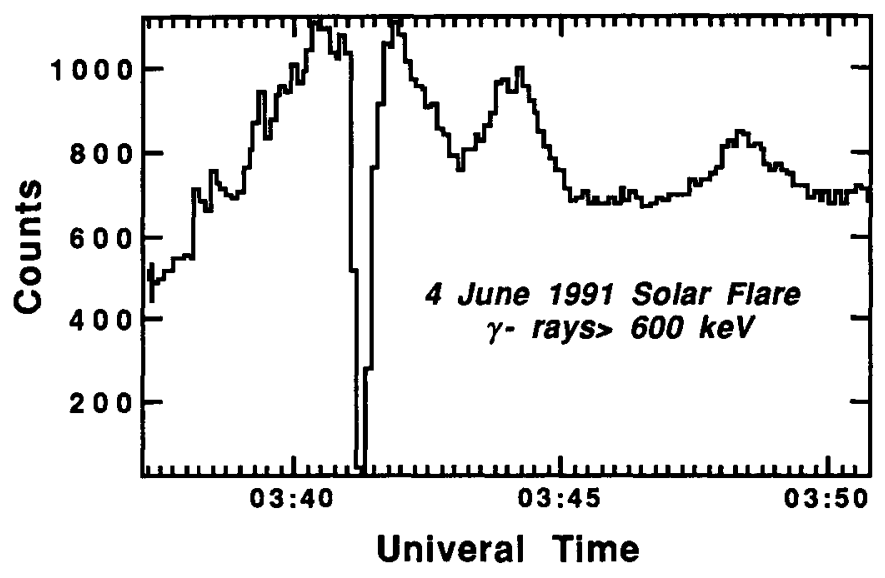

Figure 2. The uncorrected high range COMPTEL burst detector count rate from the 4 June 1991 solar flare.

\section{June 1991}

The $\gamma$-ray emission from this flare was measured by COMPTEL ${ }^{3}$. The Sun was $\sim 15^{\circ}$ off the telescope axis, well within the field-of-view. Figure 3 displays selected housekeeping data from the flare on 9 June 1991.

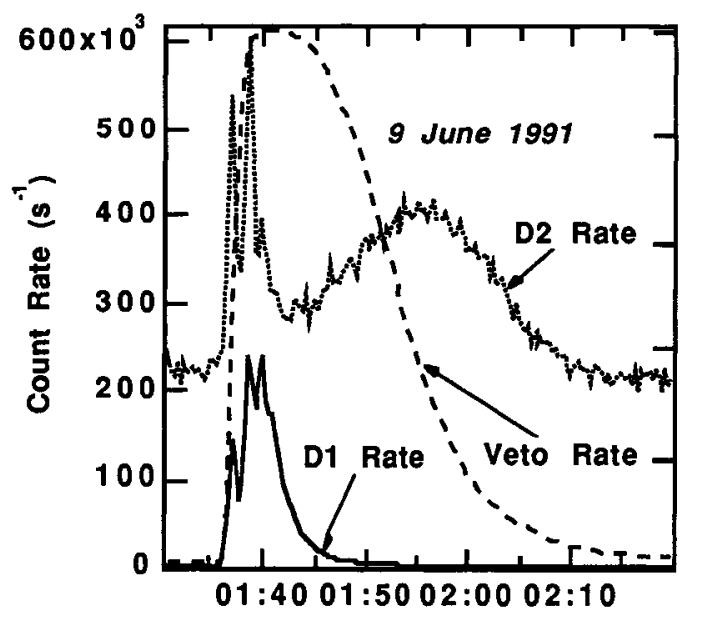

UT

Figure 3. COMPTEL housekeeping data of the flare of 9 June 1991.

This X10/3B flare was also impulsive in nature. The GOES X-ray flare started at 0134 UT peaking at 0143 UT. The $\gamma$-ray onset occurred at 0136 UT and the impulsive phase lasted until 0142 UT. Satellite sunrise precedes the flare only by a few minutes, thereby allowing a long observation of any extended flare emissions, such as neutrons. The count rates in the D1 and D2 detecting systems with 
thresholds of $\sim 60$ and $\sim 400 \mathrm{keV}$, respectively, show how the flare behaved in hard $\mathrm{X}$-rays and $\gamma$-rays. The slow rise and fall of the D2 counts rate after the impulsive phase arises from excursions in geomagnetic latitude and is not related to the flare. Also shown is the count rate in the Anticoincidence detection system. These detectors reject charged cosmic ray particles but are also sensitive to the thermal Xray flux. At the $X 10$ level, the $X$-ray flux falling upon the large Anticoincidence detectors results in pulse pile-up and large dead time effects for the whole instrument. The large dead time effects persist at least until $0155 \mathrm{UT}$, where the dead time from the Anticoincidence system drops to the $\sim 50 \%$ level. From the $\gamma$-ray events in the telescope mode, although not shown here, an image of the sun can be constructed ${ }^{3}$.

During the impulsive phase the $\gamma$-ray emission spectrum is obtained by selecting the telescope events consistent with the Sun's location, as shown in Figure 4. The strong line from deuterium formation is seen at $2.2 \mathrm{MeV}$. Other lines are also present. No background spectrum has been subtracted, but few photons are expected, since the background is suppressed by the large dead time factor making the signal to noise ratio large.

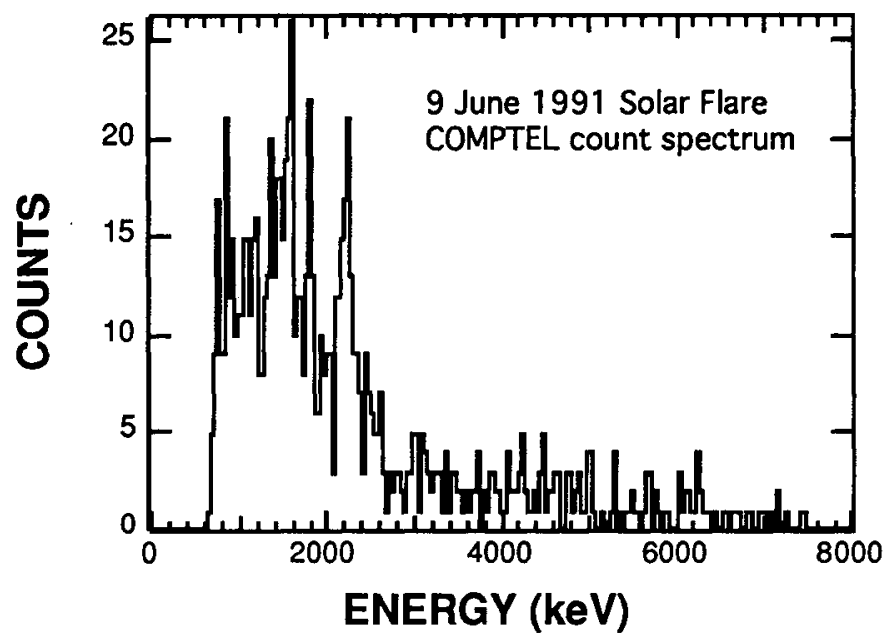

Figure 4. The raw telescope mode count spectrum from the 9 June 1991 solar flare.

Solar neutron events from 0155 UT to 0222 UT were selected from the data and reprocessed. This time selection avoids the troublesome period around the impulsive phase with large dead times and other instrumental effects. The time 0155 UT corresponds to a $50 \mathrm{MeV}$ neutron produced at the flare start $0136 \mathrm{UT}$ or a $60 \mathrm{MeV}$ neutron from the flare maximum at 0139 UT. Some selection bias, therefore, exists for neutrons detected shortly after 0155 UT (real time). The selected data were subjected to similar geometric constraints as were the $\gamma$-rays, i.e., that the origination direction of the neutron event must be consistent with the solar direction $\left( \pm 10^{\circ}\right)$. The scatter angle $(\phi)$ of the individual neutron events was restricted to $20^{\circ}$ to improve the signal-to-noise ratio. The energy of each neutron is used to compute its production time at the Sun. Shown in Figure 5 is the live time corrected and background subtracted intensity-time profile of the neutrons as produced at the Sun but plotted at the production time plus the light travel time over $1 \mathrm{AU}(507 \mathrm{~s}$ ). 
Therefore, photons and neutrons produced simultaneously are plotted at the same time value even though the neutrons arrive later.

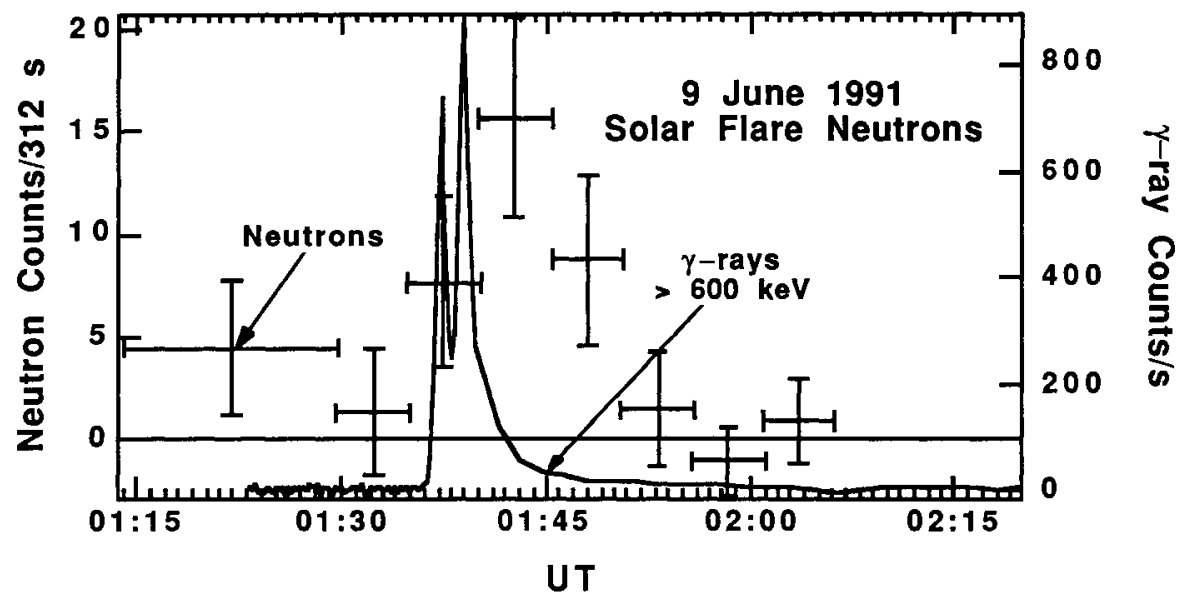

Figure 5. Neutron and $\gamma$-ray emission-time profiles for the 9 June 1991 solar flare, plotted at the time corresponding to a photon arrival time.

The background was estimated from the measured neutron flux $\sim 24$ hours later when the same orbital-geophysical conditions were reproduced. The on-board instrument software, however, was configured differently, being less restrictive in accepting neutron events. This resulted in a uniform $36 \%$ increase in the neutron background count rate. The background rate was scaled downward by this factor in order to provide a representative background for the flare orbit on 9 June. The background corrections were successfully tested on similar background orbits to ensure that a null result is obtained. Identical data cuts then were made on both the flare data and the prescaled background data. Live time fractions of $60 \%$ to $100 \%$ were applied to the data as a function of real time from 0155 - 0220 UT.

The most important feature to note in Figure 5 is the time coincidence of the onsets of neutron and the $\gamma$-ray production. The neutron intensity-time profile is expected to be smoother and somewhat broader than that of $\gamma$-rays because of the neutron energy resolution which maps into an error (FWHM) in production time at the Sun ( 1-3 minutes, dependent on energy). Although the $\gamma$-rays and neutrons are observed to start simultaneously, there is evidence that the neutron emission persists for $\sim 10$ minutes after the $>600 \mathrm{keV} \gamma$-ray flux has subsided.

This extended emission is evidence for a significant evolution (hardening) of the progenitor proton spectrum, arising from either additional acceleration or differential trapping and precipitation of the protons. As seen in Figure 4 a large fraction of the $\gamma$-rays $>1 \mathrm{MeV}$ are of nuclear origin. With no evolutin of the proton spectrum, we would expect the $>600 \mathrm{keV} \gamma$-ray flux to follow the neutron production profile. However, a hardening of the spectrum would enhance the neutron emissivity with respect to that of the nuclear $\gamma$-rays. It should be noted, though, that the primary electron bremsstrahlung component has not yet been separated from the nuclear component, so that part of the decay of the flux $>600 \mathrm{keV}$ could result from the decay of the pure electron component of the $\gamma$-ray flux. 
The trapping scenario is consistent with the $>50 \mathrm{MeV} \gamma$-ray flux detected by EGRET after $0145 \mathrm{UT}^{4}$. The net neutron count rate from $0136 \mathrm{UT}$ to $0150 \mathrm{UT}$ is positive at the $4.2 \sigma$ significance level.

\section{June 1991}

This flare also occurred in region 6659 at heliographic coordinates N31W17. As with the 9 June solar flare this flare occurred shortly after satellite sunrise within the COMPTEL field-of-view giving the longest available period of $\gamma$-ray and neutron measurements. This event like the others produced intense emission of $\gamma$-rays. The impulsive phase extended from 0158 UT until approximately 0210 UT, after which there was emission lasting until at least $0225 \mathrm{UT}$. The background subtracted burst mode intensity-time profile is shown in Figure 6a. In Figure $6 \mathrm{~b}$ is the integrated count rate (not photon) spectrum from the telescope mode clearly showing a strong $2.2 \mathrm{MeV}$ emission line and detection of photons up to $\sim 7 \mathrm{MeV}$.

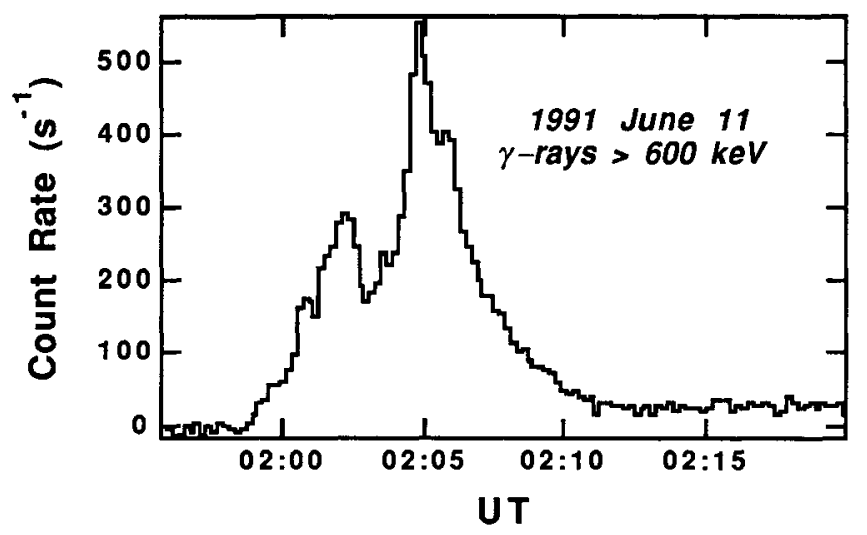

Figure 6a. The intensity-time profile of the 11 June 1991 solar flare measured by the COMPTEL burst spectrometer $(>600 \mathrm{keV})$.

Kanbach et al. $^{5}$ reported that this flare emitted $\gamma$-radiation $>50 \mathrm{MeV}$ for a period of $\sim 10$ hours with the radiation decaying away with a time constant of $\sim 25$ minutes for 2 hours following the flare, after which the flux then decayed more slowly with a time constant of 4.5 hours. As with the EGRET observations, COMPTEL measures prolonged $\gamma$-ray emission as evidenced by the image of the Sun during the first post-flare orbit using all data in the narrow $2.2 \mathrm{MeV}$ band (Figure 7).

In Figure 8 we show the intensity-time profile of the $2.2 \mathrm{MeV}$ emission during the flare orbit having selected data consistent with the solar position ${ }^{6}$. The impulsive phase is excluded from this diagram. The thermal $X$-ray flux and the intense $\gamma$-ray flux make the estimates of the $2.2 \mathrm{MeV}$ count rate highly uncertain before $0213 \mathrm{UT}$. The emission at $2.2 \mathrm{MeV}$ falls off with a decay time of $\sim 10$ minutes. The $>50 \mathrm{MeV}$ flux decay constant of 25 minutes has a large uncertainty and should be considered an upper limit ${ }^{7}$. The 10 minute decay constant measured here for $2.2 \mathrm{MeV} \gamma$-rays may, therefore, be consistent with this number.

The high energy radiation seen by EGRET $>50 \mathrm{MeV}$ and the $2.2 \mathrm{MeV}$ emission seen by COMPTEL both falling off with similar time constants point toward a common origin, i.e. a nuclear origin for both forms of the $\gamma$-radiation. If this is so, then the $>50 \mathrm{MeV}$ radiation stems from pion decay (charged and neutral) produced 
by nuclear interactions and not by primary electron bremsstrahlung as concluded by Mandzhavidze and Ramaty ${ }^{8}$. The neutrons which also arise from these interactions manifest in the deuterium formation line at $2.2 \mathrm{MeV}$. It should be noted that the time scale for the thermalization and capture of the neutrons $(\sim 100 \mathrm{~s})$ is short compared to the time constants of the $\gamma$-ray emission $(\sim 600 \mathrm{~s})$, so that the $2.2 \mathrm{MeV}$ flux represents the "instantaneous" neutron production rate.

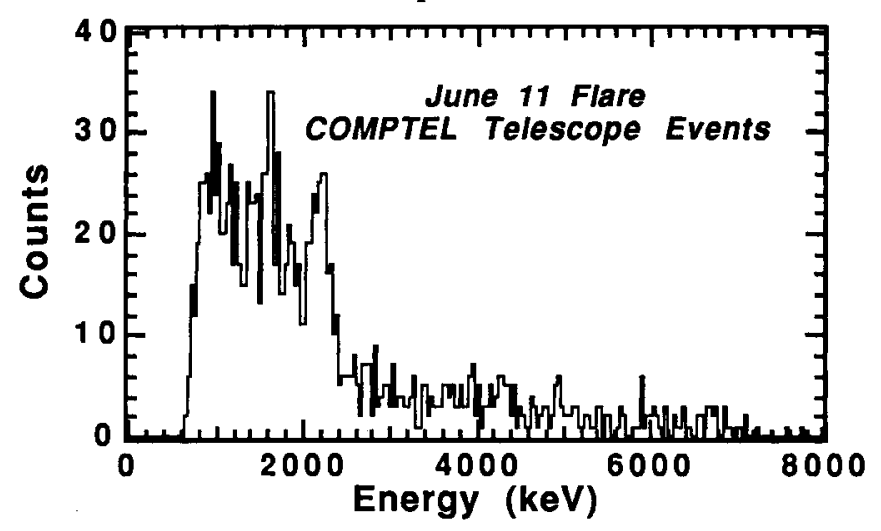

Figure $6 \mathrm{~b}$. The time integrated count spectrum measured in the telescope mode.

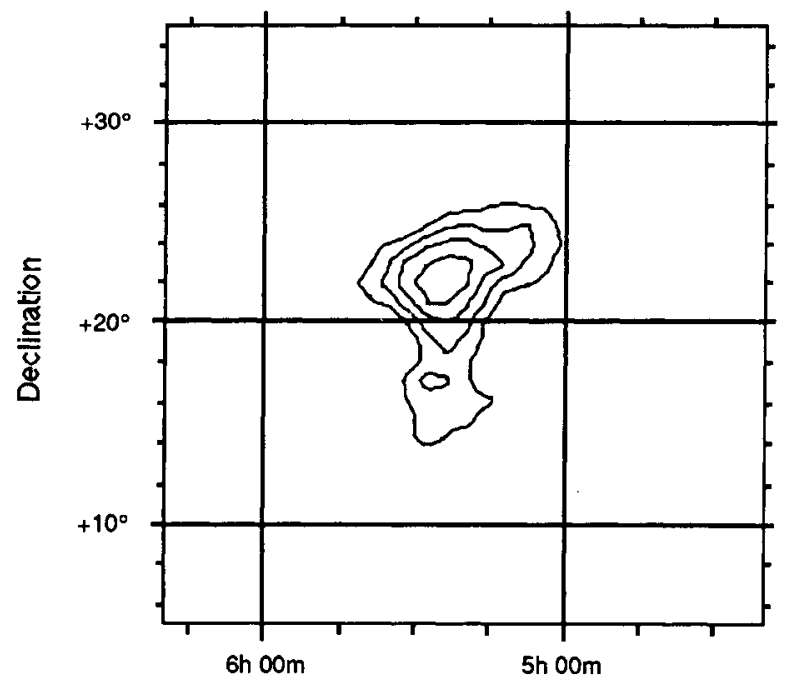

Right Ascension

Figure 7. The COMPTEL image of the Sun in the emission around the $2.2 \mathrm{MeV}$ line, from data later than $\sim 0315 \mathrm{UT}$. 


\section{June 1991}

The $\mathrm{X} 12+$ solar flare on 15 June was located at N33W69, peaking in X-rays around 0820 UT. During this time the Compton Observatory was behind the earth unable to measure the solar $\gamma$-ray flux. Upon entering daylight, the spacecraft found itself in the South Atlantic Anomaly (SAA). Only after exiting the SAA was COMPTEL able to acquire data on the remaining flare emission. This flare was also measured by the GAMMA-1 instrument ${ }^{9}$ and was reported to have emission $>30$ $\mathrm{MeV}$ after $\sim 0837 \mathrm{UT}$ lasting for at least 20 minutes after the $\mathrm{X}$-ray maximum. The COMPTEL measurements began at $\sim 0859$ UT, while the GAMMA-1 measurements ended at 0902 UT. The COMPTEL instrument continued making measurements until 0930 UT. During this time there was significant emission in the $\mathrm{MeV}$ energy range ${ }^{3}$ declining in intensity with a decay time of $830 \pm 100 \mathrm{~s}$ as shown in Figure 9.

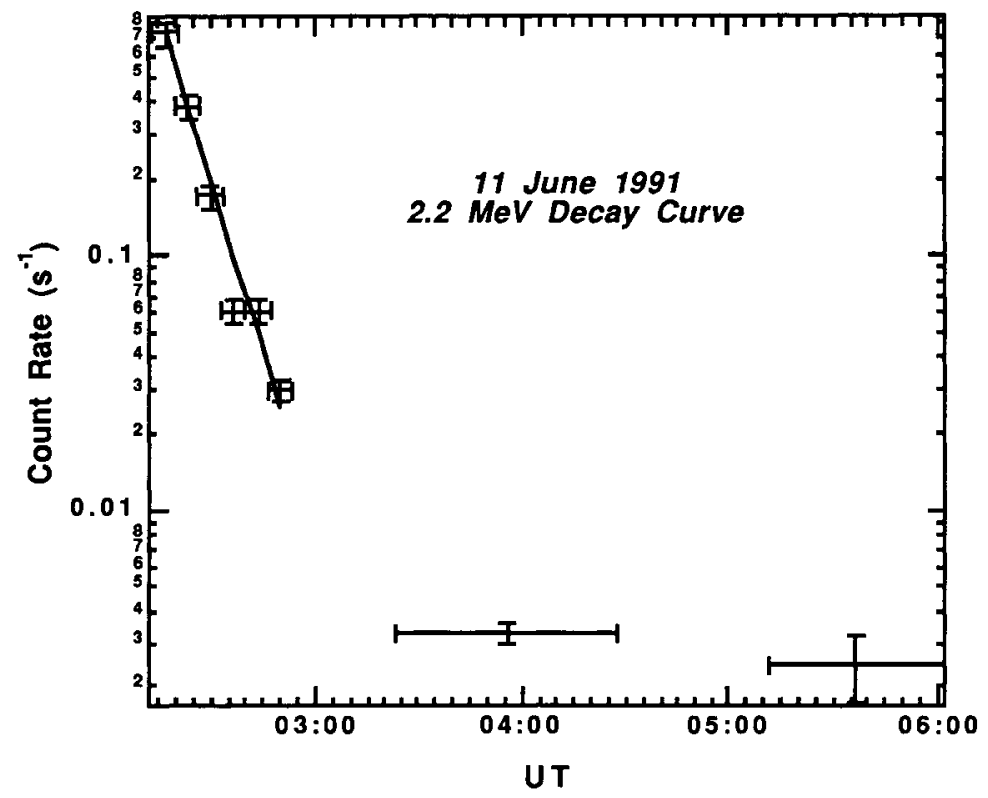

Figure 8. The count rate of the background subtracted $2.2 \mathrm{MeV}$ emission from the 11 June 1991 solar flare. Also shown is the best exponential fit to the decay of the line in the flare orbit (10.2 minutes).

The $\gamma$-ray spectrum (or $2.2 \mathrm{MeV} / 4-7 \mathrm{MeV}$ emission ratio) is typical of many solar flares in which the impulsive phase was observed indicating that, at least up to 40 minutes after the flare, there was no appreciable change in the shape of accelerated proton spectrum. Neutrons were also measured during this time ${ }^{10}$ and reflect nuclear activity at times before the $\gamma$-ray emission measured by COMPTEL, but coincident with the GAMMA-1 measurements. In Figure 10 the measured intensity-production time profile of the detected neutrons is background corrected.

As with Figure 5, the points are plotted at the arrival time of photons created simultaneously with the neutrons. The curve suffers from velocity dispersion effects, in that the counts at the earliest times in the plot are the slowest neutrons, while the counts at the later times come from higher energy neutrons. The time intervals do not sample uniformly the available neutron energy spectrum. The plot does show, 
however, that neutron emission occurred at times near the $\mathrm{X}$-ray maximum and extended for over an hour. This coupled with the detection of $\gamma$-rays up to 0930 UT means that proton acceleration or precipitation persisted for at least 70 minutes.

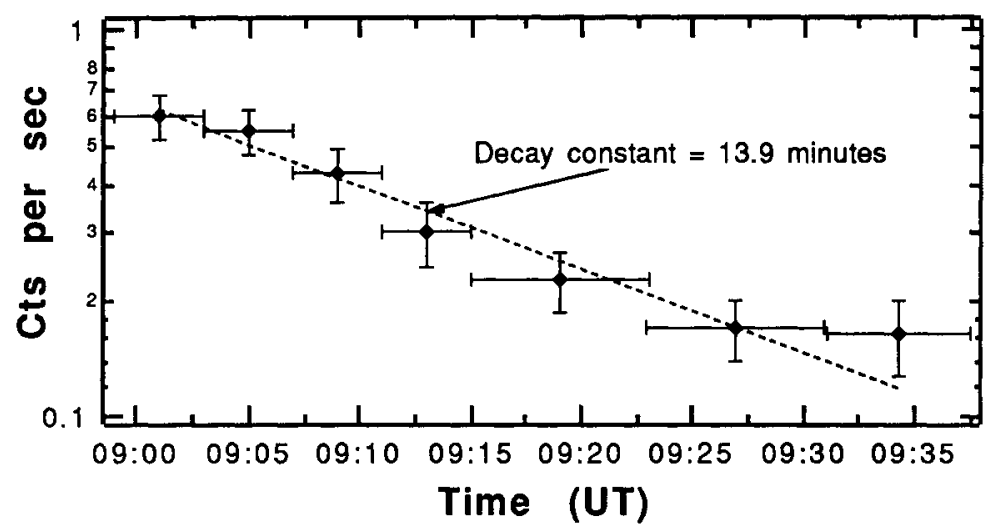

Figure 9. The $\gamma$-ray intensity-time profile of the 15 June 1991 solar flare for energies from $1-10 \mathrm{MeV}$. The decay is fit to a single exponential decay with time constant $830 \pm 100 \mathrm{~s}$.

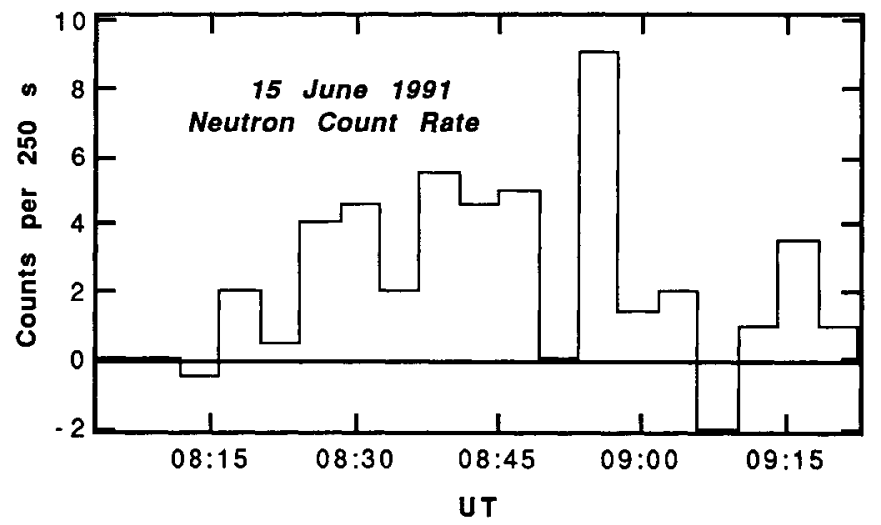

Figure 10. The background subtracted neutron production-time profile from the 15 June 1991 solar flare.

The validity of the neutron data is demonstrated by the neutron image of the Sun produced from the full neutron data set $(0859-0930 \mathrm{UT})^{10}$. The neutron events consistent with the solar location in the image are the ones used to construct the production-time profile in Figure 10.

30 June 1991

This flare occurred well outside the field-of-view of COMPTEL ( $70^{\circ}$ off axis), but was successfully measured in the burst mode. The background subtracted intensity-time profile is shown in Figure 11. 


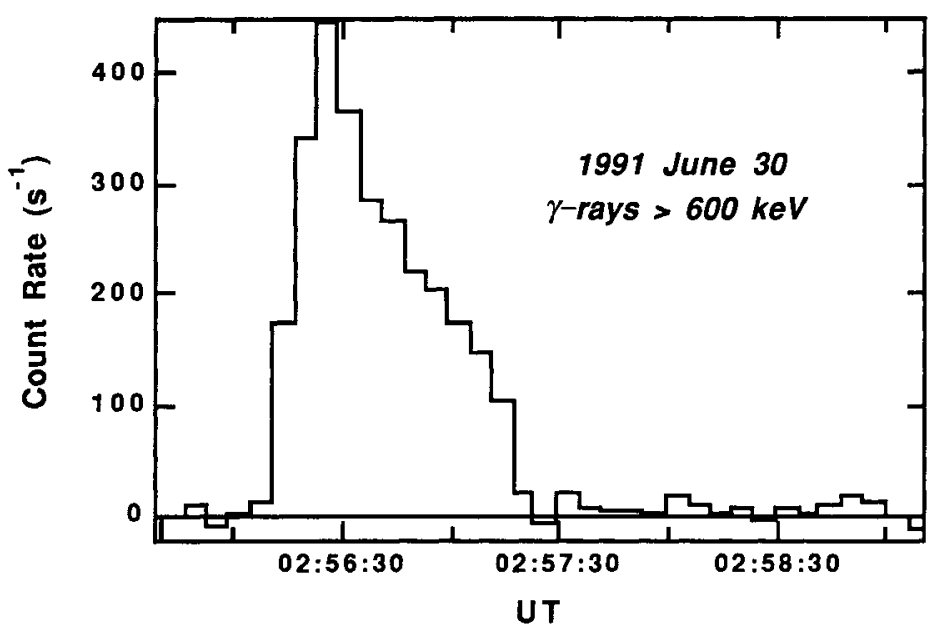

Figure 11. The background subtracted intensity-time profile for the 30 June 1991 solar flare.

The background subtracted-integrated count rate spectrum is plotted in Figure 12 , indicating emission beyond the nuclear part of the spectrum. We suspect that this indicates there was a significant number of primary electrons $>10 \mathrm{MeV}$ radiating via bremsstrahlung to produce the intensity at the higher end of the spectrum.

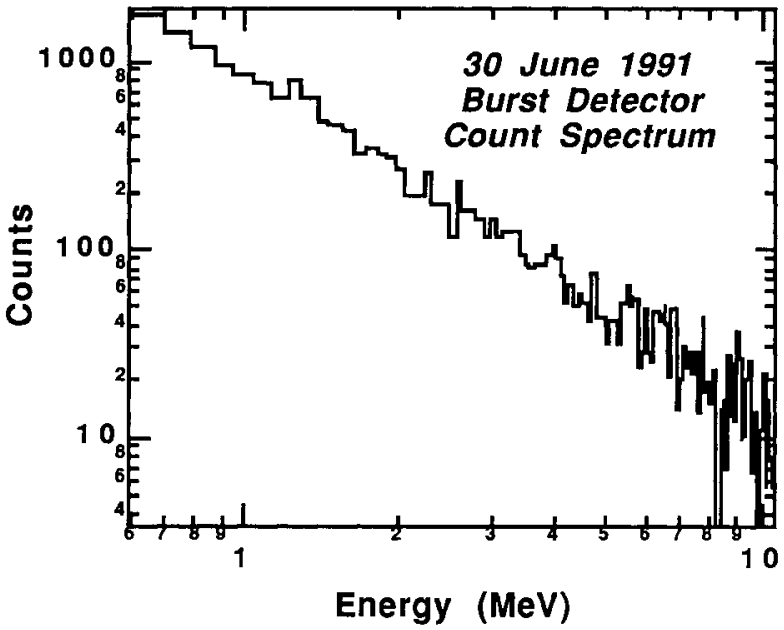

Figure 12. The count rate spectrum from the solar flare of 30 June 1991. 


\section{CONCLUSIONS}

Several large and intense events were observed by COMPTEL during June 1991 in both the telescope and burst modes. We have evidence of extended emissions of $\gamma$-rays and neutrons on a time scale of hours after the $X$-ray maximum. From the 9 June 1991 flare we have neutron evidence for prolonged high energy emission from a flare which would otherwise have been considered impulsive. We have evidence of significant proton spectral evolution (hardening) in two flares $(9,11$ June 1991) while the data of the 15 June 1991 flare shows no such evolution. We have produced the first $\gamma$-ray and neutron images of the Sun, demonstrating the high signal-to-noise ratio of the measurements. The intensity of the flares, the location of the Sun far away from the telescope axis, and the incomplete knowledge of the neutron response function mean that there is much work to be done in removing instrumental effects from the data. The final results should illuminate the phenomena reported above, yielding insights into the nature of proton acceleration and storage in the solar atmosphere.

\section{REFERENCES}

${ }^{1}$ V. Schönfelder et al., Astrophys. J. (Suppl.) accepted for publication (1992).

${ }^{2} \mathrm{C}$. Winkler et al., Adv. Space Res. 6 113-117 (1986).

${ }^{3} \mathrm{M}$. McConnell et al., Adv. Sp. Res., to be published (1992).

${ }^{4} \mathrm{G}$. Kanbach (private communication).

${ }^{5}$ G. Kanbach et al., Astron. Astrophys. in press (1992).

${ }^{6} \mathrm{G}$. Rank et al., Compton Centennial Symposium Proc., to be published (1992).

${ }^{7} \mathrm{G}$. Kanbach (private communication).

${ }^{8}$ N. Mandzhavidze and R. Ramaty, Astrophys. I. (Lett.) (1992).

${ }^{9}$ V. V. Akimov et al., in Proceedings of the 22nd International Cosmic Ray

Conference, (Dublin, Ireland, 1991), $3,73$.

${ }^{10}$ J. Ryan et al., Adv. Sp. Res., to be published (1992). 